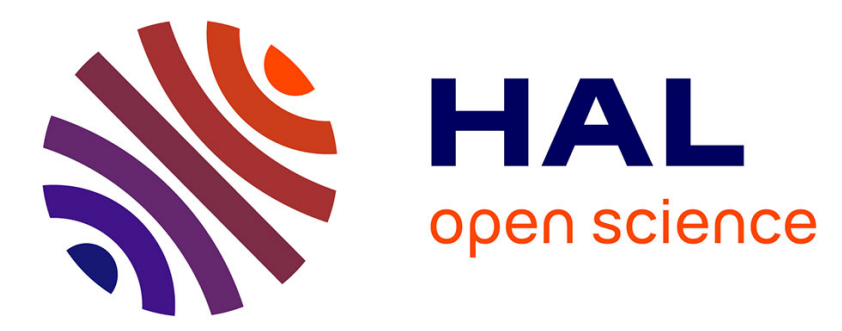

\title{
Kerr and four-wave mixing spectroscopy at the band edge of one-dimensional photonic crystals
}

Luca Razzari, Denis Träger, Magali Astic, Philippe Delaye, Robert Frey, Gérald Roosen, Régis André

\section{- To cite this version:}

Luca Razzari, Denis Träger, Magali Astic, Philippe Delaye, Robert Frey, et al.. Kerr and four-wave mixing spectroscopy at the band edge of one-dimensional photonic crystals. Applied Physics Letters, 2005, 86, pp.231106. 10.1063/1.1944887 . hal-00671148

HAL Id: hal-00671148

https://hal-iogs.archives-ouvertes.fr/hal-00671148

Submitted on 16 Feb 2012

HAL is a multi-disciplinary open access archive for the deposit and dissemination of scientific research documents, whether they are published or not. The documents may come from teaching and research institutions in France or abroad, or from public or private research centers.
L'archive ouverte pluridisciplinaire HAL, est destinée au dépôt et à la diffusion de documents scientifiques de niveau recherche, publiés ou non, émanant des établissements d'enseignement et de recherche français ou étrangers, des laboratoires publics ou privés. 


\title{
Kerr and four-wave mixing spectroscopy at the band edge of one-dimensional photonic crystals
}

\author{
Luca Razzari, ${ }^{\text {a) }}$ Denis Träger, ${ }^{\text {b) }}$ Magali Astic, Philippe Delaye, \\ Robert Frey, ${ }^{c)}$ and Gérald Roosen \\ Laboratoire Charles Fabry de l'Institut d'Optique, Centre National de la Recherche Scientifique, \\ Université Paris-Sud, Centre Scientifique Paris-Sud, Bât. 503, F-91403 Orsay Cedex, France
}

Régis André

Laboratoire de Spectrométrie Physique, Centre National de la Recherche Scientifique, Université Joseph Fourier-Grenoble, B.P. 87, F-38402 Saint Martin d'Hères Cedex, France

(Received 7 February 2005; accepted 2 May 2005; published online 3 June 2005)

\begin{abstract}
Kerr and four-wave mixing spectroscopy is shown to be a powerful technique to quantify the strong enhancement of the third-order optical nonlinear susceptibilities at the band edge of photonic crystals. Local field factors of about 5 are demonstrated for crossed Kerr effect and a narrow resonance peak observed for the conjugate reflectivity. Moreover, a reduction of the effective nonlinear susceptibility of the four-wave mixing process with increasing pump intensities is measured, which is due to different Kerr-induced blueshifts of the band edge for forward and backward pump beams and signal and conjugate beams. This observation definitely demonstrates the need for considering all the nonlinear processes for the optimization of nonlinear photonic crystals for a given application in optical signal processing. (C) 2005 American Institute of Physics.
\end{abstract} [DOI: 10.1063/1.1944887]

Due to their potential use in the development of new devices for optical signal processing, photonic crystals (PC) are presently widely studied both from the fundamental and applicative points of view. ${ }^{1,2}$ Besides passive circuits, PC microchips will need the realization of active functions such as optical switching. With respect to this need, third-order nonlinear optics will play a crucial role for the generation of such active functions. ${ }^{3-7}$ The challenge consists of the development of microsized nonlinear functions with low power consumption. However, using presently available nonlinear materials this can be achieved only by optimizing the nanoengineering of the materials in order to strongly enhance the global third-order nonlinearity. A simple concept has been proposed in order to obtain such a giant enhancement of the optical nonlinearities: The use of the PC near its band edge or at a defect in order to benefit from light localization. Indeed, in such a case, generalizing the local field theory of nonlinear homogeneous media ${ }^{8}$ to the case of photonic crystals ${ }^{9}$ shows that nonlinear susceptibilities of the PC media are enhanced by local field factors for each beam interacting in these nonlinear media. Then, the third-order nonlinear susceptibility $\chi_{\mathrm{PC}}^{(3)}$ of the $\mathrm{PC}$ is given by the expression

$$
\chi_{\mathrm{PC}}^{(3)}=f_{1} f_{2} f_{3} f_{4} \chi^{(3)},
$$

where $\chi^{(3)}$ is the intrinsic third-order susceptibility of the PC media and $f_{i}(i=1,4)$ are the local field factors for the four waves involved in the considered third-order nonlinear process. As a consequence if light localization is obtained even with a small amplitude enhancement $\left(f_{i} \approx 5\right)$, the PC nonlin-

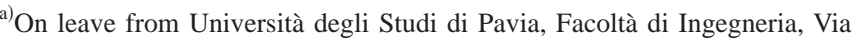
Ferrata 1, 27100 Pavia, Italy.

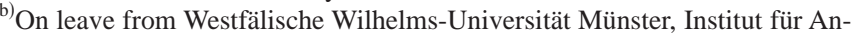
gewandte Physik, Corrensstr. 2, Universität Münster, 48149 Münster, Germany.

${ }^{c)}$ Electronic mail: robert.frey@iota.u-psud.fr
}

ear susceptibility is 625 times higher than for the same homogenous medium, which indicates that efficient small devices could actually be obtained. However detailed theoretical and experimental studies must be performed in order to demonstrate these potentiality and to define the best geometry to be chosen for the nanostructure for a given application. For this purpose, several theoretical studies have already been published ${ }^{10-12}$ but only some work has been done on the experimental side. ${ }^{13-15}$

In this letter, we focus on Kerr and four-wave mixing processes at the band edge of one-dimensional PC. Since these nonlinear optical processes which could find applications in optical signal processing are automatically phase matched, ${ }^{8}$ they make possible to perform a spectroscopic study of the corresponding optical nonlinearities and to derive useful consequences for the optimization of nanostructures for a given nonlinear function.

The sample used in this study consisted of two superposed $\mathrm{Cd}_{0.75} \mathrm{Mn}_{0.25} \mathrm{Te}-\mathrm{Cd}_{0.40} \mathrm{Mg}_{0.60}$ Te semiconductor quarterwave stacks [see Fig. 1(a)]. The back stack (20 pairs deposited on a CdMnTe buffer first deposited on the CdZnTe substrate) was designed to reflect the incident light transmitted by the front stack (30 pairs) whose band edge was therefore exactly at the center wavelength of the back Bragg mirror. The refractive indices were $n_{1}=3.088$ and $n_{2}=2.585$ for $\mathrm{Cd}_{0.75} \mathrm{Mn}_{0.25} \mathrm{Te}$ and $\mathrm{Cd}_{0.40} \mathrm{Mg}_{0.60} \mathrm{Te}$, respectively. The thicknesses of $\mathrm{Cd}_{0.75} \mathrm{Mn}_{0.25} \mathrm{Te}\left(e_{1}\right.$ and $\left.e_{1}^{\prime}\right)$ and $\mathrm{Cd}_{0.40} \mathrm{Mg}_{0.60} \mathrm{Te}\left(e_{2}\right.$ and $e_{2}^{\prime}$ ) layers were $e_{1}=54 \mathrm{~nm}$ and $e_{2}=64.6 \mathrm{~nm}$ for the front stack (the studied one-dimensional PC) and $e_{1}^{\prime}=57.3 \mathrm{~nm}$ and $e_{2}^{\prime}=68.5 \mathrm{~nm}$ for the back Bragg mirror. This design was chosen to automatically create the backward pump beam at the band edge of the PC for the four-wave mixing process. As a consequence of this design, for a lossless photonic crystal all the beams would be totally reflected for wavelengths inside the band gap of the PC or of the back Bragg mirror. As seen in Fig. 1(b), this is not the case at low photon wavelengths 


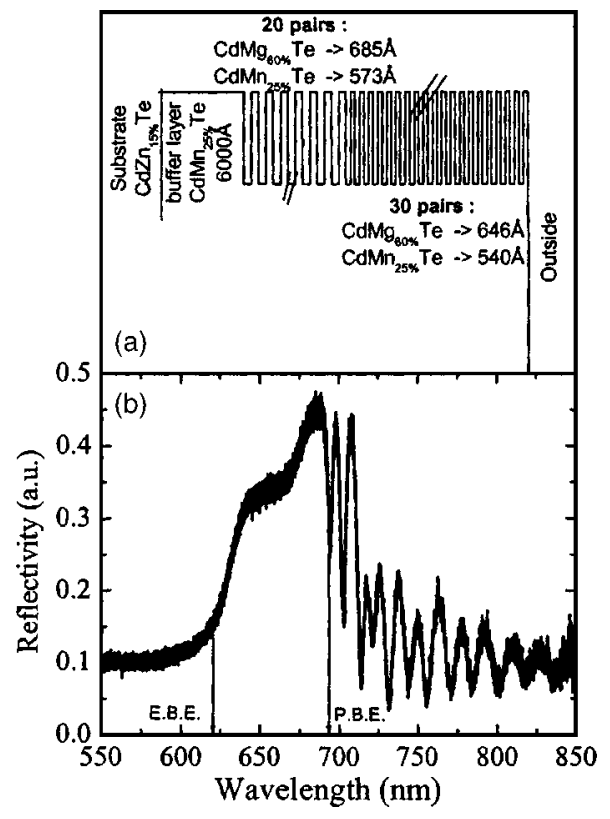

FIG. 1. Sketch of the sample plotted on a refractive index scale (a) and reflectivity spectrum of the structure at normal incidence (b). EBE and PBE stand for electronic and photonic band edges, respectively.

where band absorption occurs at the electronic band edge (EBE), of $\mathrm{Cd}_{0.75} \mathrm{Mn}_{0.25}$ Te located at $620 \mathrm{~nm}$ and lowers the reflectivity. Strong losses occur also even below this EBE at the photonic band edge (PBE) of the PC (see the deep hole marked by the arrow in the reflectivity spectrum) due to the local field enhancement of the very small diffusion losses $(0.07 \%)$ occurring at the layer interfaces. It is interesting, by the way, to note that this technique could be used to quantify such losses which could be very difficult to perform otherwise.

For the nonlinear experiments, we used picosecond duration frequency tunable laser pulses produced by optical parametric generation (OPG) and amplification (OPA) in BBO crystals. OPG and OPA were obtained using a frequency doubled mode-locked Nd-YAG laser as the pump beam. The choice of a picosecond duration for the laser pulses resulted from the compromise between the use of the highest beam intensity for a given pulse energy and the wavelength selectivity necessary to resolve the band edge zone of the PC. The beam delivered by the OPG-OPA was separated into two beams. The most intense one was sent perpendicularly to the PC and acted as the forward pump beam. The backward pump beam was generated by reflexion of the forward pump beam on the back Bragg mirror of the sample. The low intensity signal beam was sent at the small incidence angle $\theta=2^{\circ}$ and the conjugated beam was generated exactly in the opposite direction to the signal beam. The intensities of all beams were measured in order to characterize the nonlinear behavior of the PC.

Spectra of pump and signal reflectivities and phase conjugate reflectivity were recorded for different pump intensities. As an example, Fig. 2 shows such spectra recorded for $I_{p}=0.22 \mathrm{GW} / \mathrm{cm}^{2}$ and $I_{p}=0.50 \mathrm{GW} / \mathrm{cm}^{2}$. In Figs. 2(a) and 2(b), the pump reflectivity (stars) and the signal reflectivity without and with the pump pulse present (closed and open triangles respectively) are plotted as a function of the laserwavelength. The phase conjugate spectra appear in Figs. 2(c) and 2(d). As demonstrated in Figs. 2(a) and 2(b), the PC

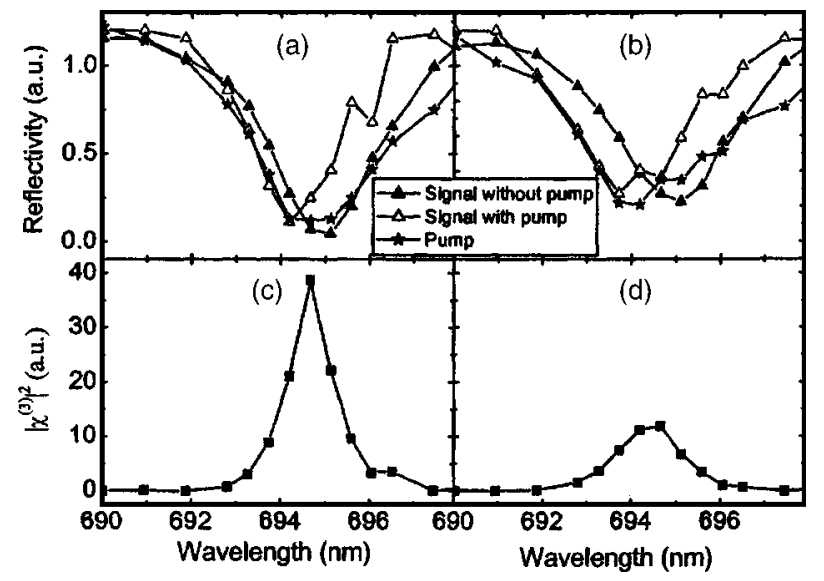

FIG. 2. Pump and signal, and conjugated reflectivity spectra [(a) and (b), and (c) and (d), respectively]. (a) and (c) correspond to spectra recorded for pump intensities of $0.22 \mathrm{GW} / \mathrm{cm}^{2}$ whereas (b) and (d) were recorded for pump intensities of $0.50 \mathrm{GW} / \mathrm{cm}^{2}$.

band edge represented by the hole in the reflectivity spectra is blueshifted for both signal and pump beams when the pump pulse is present on the sample. Moreover, this blueshift is almost proportional to the pump intensity $\left(\Delta \lambda_{P}=0.3 \mathrm{~nm}\right.$ and $0.4 \mathrm{~nm}$ and $\Delta \lambda_{S}=0.5 \mathrm{~nm}$ and $0.8 \mathrm{~nm}$ with a precision of $\pm 0.1 \mathrm{~nm}$ for $I_{p}=0.22 \mathrm{GW} / \mathrm{cm}^{2}$ and $I_{p}=0.50 \mathrm{GW} / \mathrm{cm}^{2}$, respectively). It must also be stressed that within the experimental errors the signal blueshift is twice that experienced by the pump beam due to the degeneracy of Kerr effect in the latter case. ${ }^{8}$ From these blueshifts, it is possible to evaluate the refractive index change by using the expression of the band edge wavelength $\lambda_{B}$ given in Ref. 16 .

$$
\lambda_{0} / \lambda_{B}=\arccos \left[-T_{12} \cos (\pi / N)+R_{12}\right],
$$

where $\lambda_{0}=4 n_{1} e_{1}=4 n_{2} e_{2}$ is the center wavelength of the band gap, and $T_{12}=4 n_{1} n_{2} /\left(n_{1}+n_{2}\right)^{2}$ and $R_{12}=\left(n_{1}-n_{2}\right)^{2} /\left(n_{1}+n_{2}\right)^{2}$ are the transmission and reflexion coefficients of the interfaces, respectively. Indeed, at high intensities, the refractive indices are modified $\left(n_{i}=n_{i}+\Delta n_{i}\right.$ with $\Delta n_{i}$ the nonlinear refractive index change for $i=1,2) .{ }^{8}$ As $n_{1} \approx n_{2}$, the change in the band edge wavelength is mainly related to the modification of $\lambda_{0}$ and is given, in first approximation, by

$$
\Delta \lambda_{B} / \lambda_{B}=\left[\Delta n_{1} / n_{1}+\Delta n_{2} / n_{2}\right] / 2 .
$$

As the band edge wavelength of the PC is very near the electronic band gap of $\mathrm{Cd}_{0.75} \mathrm{Mn}_{0.25} \mathrm{Te}$ and far from that of $\mathrm{Cd}_{0.40} \mathrm{Mg}_{0.60} \mathrm{Te}$, only the change of $n_{1}$ is of importance for determining the optical band edge blueshift of the PC. The nonlinear refractive index change for the crossed Kerr effect is then $\Delta n=-0.007$ and $\Delta n=-0.004$ for pump beam intensities of $I_{p}=0.50 \mathrm{GW} / \mathrm{cm}^{2}$ and $I_{p}=0.22 \mathrm{GW} / \mathrm{cm}^{2}$, respectively. The corresponding third-order susceptibility is then enormous $\left(\chi_{\mathrm{PC}}^{(3)}=-1.2 \times 10^{-8} \mathrm{esu}\right)$ which explains why these large changes in reflectivity occur with only small input intensities. Moreover using the value $\chi^{(3)}=-4 \times 10^{-11}$ esu calculated for the third order nonlinear susceptibility of bulk $\mathrm{Cd}_{0.75} \mathrm{Mn}_{0.25} \mathrm{Te}$ (Ref. 17) and assuming equal local field factors for all wavelengths, Eq. (1) gives the value $f=4.2$ for the mean local field factor, in good agreement with that calculated using the transfer matrix method. ${ }^{18}$

As expected from the high value of the measured local field factor, the four-wave mixing process was strongly enhanced at the band edge of the PC. This is illustrated in Figs. 


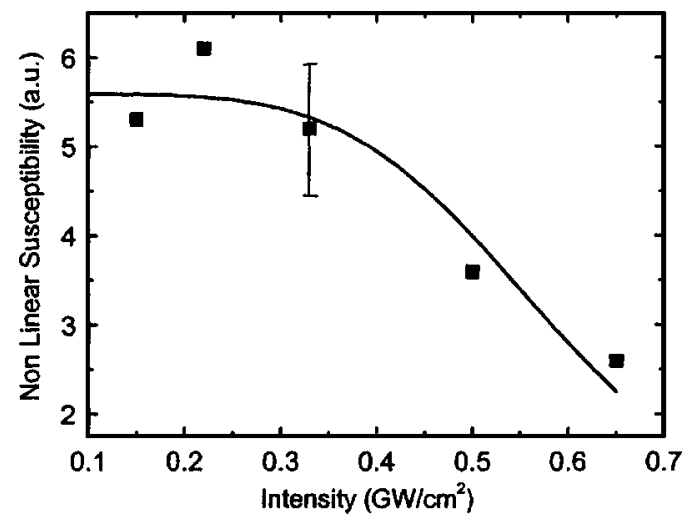

FIG. 3. Effective third-order nonlinear optical susceptibility vs the pump intensity.

2(c) and 2(d) showing the strong resonance of the phase conjugate reflectivity normalized to the pump and signal input intensities around $694 \mathrm{~nm}$. Due to the low intensities used in our experiments, it was not possible to measure the phase conjugate reflectivity outside of the band edge region of the PC. Nevertheless, the contrast is higher than one hundred which confirms the local field enhancement of the phase conjugate reflectivity. It must be stressed that the phase conjugate reflectivity spectrum widens with increasing pump intensities and that the maximum value of the measured effective nonlinear susceptibility strongly depends on the pump intensity. This last behavior is illustrated in Fig. 3 showing the peak effective third-order nonlinear susceptibility plotted as a function of the pump intensity. The value is high and constant up to $0.3 \mathrm{GW} / \mathrm{cm}^{2}$ and strongly decreases with increasing pump intensities. This is evidently connected to the Kerr-induced blueshift of the PC band edge which is two times larger for the signal than for the pump beams. As a consequence, spectra of the local field factors for forward and backward pump beams are shifted with respect to those corresponding to signal and conjugate beams, which gives rise to a less important impact of light localization on the effective nonlinear susceptibilities. This observation demonstrates that all the nonlinear processes must be taken into account in the design of the photonic crystal in order to optimize a given nonlinear process. In particular, for the four-wave mixing process considered here, the use of materials with positive nonlinear susceptibilities may produce a red Kerr shift for the band edge which could be compensated for by the blueshift experienced by the signal and conjugate beams, due to their angle of incidence on the PC.

In conclusion, Kerr and four-wave mixing spectroscopy have been used in order to experimentally quantify the strong enhancement of the corresponding third order nonlinear susceptibilities. Our results demonstrate local field factors larger than four for a low contrast one-dimensional PC and a narrow peak for the phase conjugate reflectivity due to light localization. Moreover, a reduction of the enhancement of the phase conjugate reflectivity was demonstrated at high laser intensities due to different Kerr-induced blueshifts experienced by the forward and backward pump beams on one hand and signal and conjugate beams on the other hand. This result definitely demonstrates that all the possible nonlinear processes must be taken into account for an optimized design of nonlinear photonic crystals for optical signal processing.

Two of the authors (D. T. and L. R.) were supported by the European Commission under the Marie Curie Training Site "Information Optics, New Tools and Application" (HPMT-CT-2000-00105). One of those authors (D. T.) also acknowledges support from Konrad-Adenauer-Stiftung e.V.

${ }^{1}$ R. M. De La Rue, Opt. Quantum Electron. 34, 1 (2002).

${ }^{2}$ T. F. Krauss and T. Baba, IEEE J. Quantum Electron. 38, 724 (2002).

${ }^{3}$ M. Scalora, J. P. Dowling, C. M. Bowden, and M. J. Bloemer, Phys. Rev. Lett. 73, 1368 (1994).

${ }^{4}$ S. John and T. Quang, Phys. Rev. A 54, 4479 (1996).

${ }^{5}$ P. Tran, Opt. Lett. 21, 1138 (1996).

${ }^{6}$ M. Soljacic, M. Ibanescu, S. G. Johnson, Y. Fink, and J. D. Joannopoulos, Phys. Rev. E 66, 055601 (2002).

${ }^{7}$ A. Forschel, Nat. Mater. 2, 13 (2003).

${ }^{8}$ Y. R. Shen, The Principles of Nonlinear Optics (Wiley, New York, 1984); R. W. Boyd, Nonlinear Optics (Academic, New York, 2003).

${ }^{9}$ R. Frey, P. Delaye, and G. Roosen, Nanophotonique, edited by J-M. Lourtioz, C. Delalande, A. Levenson, and H. Rigneault (Hermès-Lavoisier, Paris, 2005).

${ }^{10}$ P. Tran. J. Opt. Soc. Am. B 14, 2589 (1997).

${ }^{11}$ R. L. Nelson and R. W. Boyd. J. Mod. Opt. 46, 1061 (1999).

${ }^{12}$ P. Xie and Z.-Q. Zhang, Phys. Rev. A 69, 053806 (2004).

${ }^{13}$ G. J. Schneider and G. H. Watson, Appl. Phys. Lett. 83, 5350 (2003).

${ }^{14}$ A. V. Andreev, A. V. Balakin, A. B. Kozlov, I. A. Ozheredov, I. R. Prudnikov, A. P. Shkurinov, P. Masselin, and G. Mouret, J. Opt. Soc. Am. B 19, 1865 (2002).

${ }^{15}$ H. Inouye and Y. Kanemitsu, Appl. Phys. Lett. 82, 115 (2003).

${ }^{16}$ J. M. Bendickson, J. P. Dowling, and M. Scalora, Phys. Rev. E 53, 4107 (1996).

${ }^{17}$ M. Sheik-Bahae, D. C. Hutchings, D. J. Hagan, and E. W. Van Stryland, IEEE J. Quantum Electron. 27, 1296 (1991).

${ }^{18}$ P. Delaye, R. Frey, M. Astic, and G. Roosen (unpublished). 